\title{
Delays in thrombolysis during COVID-19 are associated with worse neurological outcomes: the Society of Vascular and Interventional Neurology Multicenter Collaboration
}

\author{
Dinesh V. Jillella ${ }^{1}(1) \cdot$ Fadi Nahab ${ }^{1} \cdot$ Thanh N. Nguyen $^{2} \cdot$ Mohamad Abdalkader $^{3} \cdot$ David S. Liebeskind $^{4} \cdot$ Nirav Vora $^{5}$. \\ Vivek Rai ${ }^{5}$. Diogo C. Haussen ${ }^{6} \cdot$ Raul G. Nogueira ${ }^{6}$. Shashvat Desai ${ }^{7,8}$. Ashutosh P. Jadhav ${ }^{7,8}$. Alexandra L. Czap ${ }^{9}$. \\ Alicia M. Zha ${ }^{9}$. Italo Linfante ${ }^{10}$. Ameer E Hassan ${ }^{11}$. Darko Quispe-Orozco ${ }^{12}$ - Santiago Ortega-Gutierrez ${ }^{12}$. \\ Priyank Khandelwal ${ }^{13} \cdot$ Pratit Patel $^{13} \cdot$ Osama Zaidat ${ }^{14} \cdot$ Tudor G. Jovin $^{15} \cdot$ Scott Kamen $^{16}$. James E. Siegler ${ }^{15}$
}

Received: 1 June 2021 / Revised: 21 July 2021 / Accepted: 26 July 2021 / Published online: 31 July 2021

(c) Springer-Verlag GmbH Germany, part of Springer Nature 2021

\begin{abstract}
Introduction We have demonstrated in a multicenter cohort that the COVID-19 pandemic has led to a delay in intravenous thrombolysis (IVT) among stroke patients. Whether this delay contributes to meaningful short-term outcome differences in these patients warranted further exploration.

Methods We conducted a nested observational cohort study of adult acute ischemic stroke patients receiving IVT from 9 comprehensive stroke centers across 7 U.S states. Patients admitted prior to the COVID-19 pandemic (1/1/2019-02/29/2020) were compared to patients admitted during the early pandemic (3/1/2020-7/31/2020). Multivariable logistic regression was used to estimate the effect of IVT delay on discharge to hospice or death, with treatment delay on admission during COVID19 included as an interaction term.

Results Of the 676 thrombolysed patients, the median age was 70 (IQR 58-81) years, 313 were female (46.3\%), and the median NIHSS was 8 (IQR 4-16). Longer treatment delays were observed during COVID-19 (median 46 vs 38 min, $p=0.01$ ) and were associated with higher in-hospital death/hospice discharge irrespective of admission period (OR per hour 1.08, 95\% CI 1.01-1.17, $p=0.03$ ). This effect was strengthened after multivariable adjustment (aOR 1.15, 95\% CI 1.07-1.24, $p<0.001)$. There was no interaction of treatment delay on admission during COVID-19 $\left(p_{\text {interaction }}=0.65\right)$. Every one-hour delay in IVT was also associated with $7 \%$ lower odds of being discharged to home or acute inpatient rehabilitation facility (aOR 0.93, 95\% CI 0.89-0.97, $p<0.001$ ).

Conclusion Treatment delays observed during the COVID-19 pandemic led to greater early mortality and hospice care, with a lower probability of discharge to home/rehabilitation facility. There was no effect modification of treatment delay on admission during the pandemic, indicating that treatment delay at any time contributes similarly to these short-term outcomes.
\end{abstract}

Keywords Stroke $\cdot$ COVID-19 $\cdot$ Thrombolysis $\cdot$ Treatment delay $\cdot$ Outcomes $\cdot$ Mortality

\section{Introduction}

The coronavirus disease 2019 (COVID-19) pandemic has had a deleterious impact on health care systems across the world. In addition to its direct neurological manifestations, notable indirect effects pertain to the delays in presentation and management of emergent medical conditions like

James E. Siegler

siegler-james@cooperhealth.edu

Extended author information available on the last page of the article myocardial infarction, and stroke [1-3]. Recent studies have also shown declines in stroke admissions during the pandemic, but with limited data on its impact on stroke management metrics and their related outcomes [4].

Delays in thrombolysis are associated with poor clinical outcomes with a large registry study involving 55, 296 ischemic stroke patients showing higher odds of in-hospital mortality associated with such delays [5]. Our recently published multicenter study involving high-volume stroke centers across the U.S showed significantly greater delays in reperfusion therapy with intravenous thrombolysis (IVT) during the early pandemic period compared to the pre-pandemic 
period [6]. We aimed to elucidate the impact of such delays on early neurological outcomes.

\section{Methods}

A nested cohort study of patients who received IVT and had completed data regarding door-to-needle time and symptomatic intracranial hemorrhage ( $\mathrm{sICH}$ ) within $36 \mathrm{~h}$ was conducted using a previously described observational registry $[4,6]$. Patients with stroke admitted prior to the COVID-19 pandemic (1/1/2019-02/29/2020) were compared to patients admitted during the early pandemic (3/1/2020-7/31/2020). In addition to the variables previously described $[4,6]$, sICH was collected from participating registries and defined as any clinical worsening attributed to an intracranial hemorrhage that occurred within $36 \mathrm{~h}$ of thrombolysis [7]. Based on completeness of data for this subgroup analysis, consecutive acute ischemic stroke patients admitted to 9 Comprehensive Stroke Centers in 7 U.S. states were screened for inclusion. Patients were excluded if they were not treated with IVT, if they had unavailable door-to-needle times, or incomplete documentation of sICH. None of the participating sites in this analysis utilized a mobile stroke unit. Data will be made available upon reasonable request of a qualified investigator with the approval of the local regulatory board.

Descriptive statistics were used to summarize differences in key clinical, management, and outcome variables. Categorical variables are expressed as proportions and were compared between study periods using the Chi-square test, and non-normally distributed continuous variables as medians with interquartile range, with differences assessed using the Wilcoxon Rank-Sum test.

The pre-specified primary outcome was a composite of discharge to hospice or in-hospital mortality, which was defined as an unfavorable disposition. The secondary outcome was a favorable discharge disposition (composite of discharge to home or an acute inpatient rehabilitation facility [IRF]). Unadjusted linear and logistic regression models were used to estimate the effect of covariates on the primary outcome measure. All variables significant to $p<0.1$ were then entered into two multivariable logistic regression models-one with a multiplicative interaction term of treatment delay on COVID-19 period, and one without the interaction term. The performance of each model was assessed using the Akaike Information Criterion (AIC). A multivariable regression model was also generated to estimate the effect of delay in door-to-needle time on the secondary outcome (favorable disposition). This model was derived using the aforementioned technique, with the inclusion of the interaction term (treatment delay $\times$ COVID-19). Exploratory subgroup analyses were used to confirm the effect of treatment delay on death/hospice irrespective of admission prior to
( $n=537)$ or during COVID-19 $(n=139)$ using multivariable logistic regression after adjusting for variables significant to $p<0.1$ in univariate regression, as detailed above. All multivariable models were clustered by site.

Missing data were not imputed. All tests were two-sided with a significance level set at 0.05 . No adjustments were made for multiple comparisons. $P$ values are provided for a convention but should be interpreted with caution as analyses were exploratory. All analyses were performed using STATA 15.0 (College Station, TX).

\section{Results}

Of the 6257 patients admitted during the study period, 778 received IVT $(12.4 \%)$. One hundred two patients were excluded due to unavailable door-to-needle times. Of the 676 included, the median age was 70 (IQR 58-81), 313 (46.3\%), were female, and the median NIHSS was 8 (IQR 4-16). The simplified demographic, treatment and clinical outcomes are summarized in Table 1.

There were $62(9.1 \%)$ patients out of the 676 included with an unfavorable disposition of in-hospital death or discharge to hospice during the study period. There was no significant increase in death or hospice discharge during COVID-19 when compared to the preceding period (10.1\% vs. $8.9 \%, p=0.68)$. A majority ( $73 \%$ ) were discharged to a favorable disposition throughout the study period, with no difference across the two periods (69.8\% [COVID-19] vs. $74.1 \%$ [Pre-COVID-19], $p=0.30$ ). Patients treated during COVID-19 had a median delay of $8 \mathrm{~min}$ from door-to-needle in comparison with the preceding period (median 46 [IQR 29-64] vs. 38 [IQR 26-56], $p=0.01$ ).

\section{Primary outcome}

In univariate analysis, age, white race, atrial fibrillation, heart failure, baseline NIHSS, door-to-needle time, and sICH were associated with in-hospital death or discharge to hospice (Table 2). Every hour delay in IVT was associated with $8 \%$ higher odds of death/hospice (OR per hour 1.08 , 95\% CI 1.01-1.17, $p=0.03$ ). In the multivariable logistic regression model, including all variables significantly associated in univariate analysis, including an interaction term for treatment delay on COVID-19 period), the association between delay in IVT was strengthened, with every hour corresponding to $15 \%$ higher odds of discharge to hospice/ death (aOR 1.15, 95\% CI 1.07-1.24, $p<0.001$ ). There was no significant interaction between treatment delay and admission during the COVID-19 period on the outcome of death/hospice in this model ( $p=0.65$ for interaction). Model performance was similar with and without the interaction term (AIC 284.28 vs. 284.31). In subgroup analyses, after 
Table 1 Demographic and clinical differences between study periods

\begin{tabular}{|c|c|c|c|}
\hline & $\begin{array}{l}\text { Jan 2019-Feb } 2020 \\
(n=537)\end{array}$ & $\begin{array}{l}\text { Mar 2020-July } 2020 \\
(n=139)\end{array}$ & $p$ value \\
\hline Age, median y (IQR) & $71(59-82)$ & $68(57-77)$ & 0.05 \\
\hline Female sex, no. $(\%)$ & $251(46.7 \%)$ & $62(44.6 \%)$ & 0.65 \\
\hline Race, no. $(\%)$ & & & $<0.01$ \\
\hline White & $412(76.7 \%)$ & $88(63.3 \%)$ & \\
\hline Black & $88(16.4 \%)$ & $40(28.8 \%)$ & \\
\hline Other/unknown & $37(6.9 \%)$ & $11(7.9 \%)$ & \\
\hline Hispanic ethnicity, no. (\%) & $180(33.5 \%)$ & $42(30.2 \%)$ & 0.46 \\
\hline Transfer from outside hospital, no. (\%) & $13(3.0 \%)$ & $8(6.0 \%)$ & 0.11 \\
\hline \multicolumn{4}{|l|}{ Past medical history, no. (\%) } \\
\hline Hypertension & $417(77.7 \%)$ & $88(63.3 \%)$ & $<0.01$ \\
\hline Dyslipidemia & $293(54.6 \%)$ & $74(53.2 \%)$ & 0.78 \\
\hline Diabetes & $186(34.6 \%)$ & $44(31.7 \%)$ & 0.51 \\
\hline Coronary artery disease & $99(18.4 \%)$ & $24(17.3 \%)$ & 0.75 \\
\hline Prior ischemic stroke & $95(17.7 \%)$ & $32(23.0 \%)$ & 0.15 \\
\hline Atrial fibrillation & $93(17.3 \%)$ & $27(19.2 \%$ & 0.56 \\
\hline Tobacco use & $69(12.9 \%)$ & $19(13.7 \%)$ & 0.80 \\
\hline Heart failure & $54(10.1 \%)$ & $20(14.4 \%)$ & 0.15 \\
\hline NIHSS, median (IQR) & $8(4-16)$ & $10(5-17)$ & 0.16 \\
\hline Proximal large vessel occlusion, no. (\%) & $127(23.7 \%)$ & $35(25.2 \%)$ & 0.71 \\
\hline Thrombectomy, no. (\%) & $106 / 127(83.5 \%)$ & $29 / 35(82.9 \%)$ & 0.93 \\
\hline Door-to-needle, median min. (IQR) & $38(26-56)$ & $46(29-64)$ & 0.01 \\
\hline sICH & $23(4.3 \%)$ & $6(4.3 \%)$ & 0.99 \\
\hline In-hospital death/discharge to hospice, no. (\%) & $48(8.9 \%)$ & $14(10.1 \%)$ & 0.68 \\
\hline Discharge to home/acute rehab, no. (\%) & $398(74.1 \%)$ & $97(69.8 \%)$ & 0.30 \\
\hline
\end{tabular}

$I Q R$ denotes interquartile range, NIHSS National Institutes of Health Stroke Scale, and $S I C H$ symptomatic intracerebral hemorrhage within $36 \mathrm{~h}$ of thrombolysis multivariable adjustment and clustering by site, delays in IVT remained independently associated with higher odds of in-hospital mortality/hospice prior to the COVID-19 pandemic (aOR 1.17, 95\% CI 1.06-1.28, $p=0.001$ ) and during the pandemic (aOR 1.10, 95\% CI 1.02-1.19, $p=0.017$ ).

\section{Secondary outcome}

With regard to the secondary outcome of favorable discharge to home or IRF, in the univariate analysis, older age, female sex, atrial fibrillation, heart failure, NIHSS, door-to-needle time per hour and sICH (all negative predictors), along with tobacco use (positive predictor) were associated with discharge to a favorable disposition of home or IRF. Longer delays to IVT remained associated with a non-significantly lower probability of discharge to a favorable discharge disposition (OR per hour $0.95,95 \%$ CI $0.89-1.007, p=0.089$ ). In multivariable model, including all variables significantly associated with favorable discharge in univariate regression, and clustering by site, every one-hour delay in IVT was associated with a $7 \%$ reduction in the odds of being discharged to home or IRF (aOR 0.93, 95\% CI 0.89-0.97, $p<0.001)$.

\section{Discussion}

In this follow-up investigation to a prior analysis which established that patients admitted during COVID-19 are treated more slowly with thrombolysis, [6] we now confirm that these delays in treatment are independently associated with worse short-term outcomes. Importantly, there was no modification of this effect by the period in which treatment was administered-e.g., treatment delays were associated with similarly higher short-term mortality/hospice rates irrespective of the treatment period (COVID-19 vs. pre-COVID-19). Moreover, the strength of the association improved after adjustment for clinically important, measurable confounders. Together, these data indicate that treatment delays during COVID-19, rather than any significant effect of the COVID-19 period itself, are important contributors to early adverse outcomes of stroke patients treated during the pandemic. Although we did not find a significant difference 
Table 2 Unadjusted and adjusted regression model for in-hospital death or discharge to hospice

\begin{tabular}{|c|c|c|c|c|}
\hline & \multicolumn{2}{|c|}{ Unadjusted regression model } & \multicolumn{2}{|c|}{ Adjusted regression model ${ }^{\mathrm{a}}$} \\
\hline & OR (95\% CI) & $p$ value & OR $(95 \% \mathrm{CI})$ & $p$ value \\
\hline COVID-19 admission & $0.88(0.47-1.64)$ & 0.68 & & \\
\hline Door-to-needle, per hour & $1.08(1.01-1.17)$ & 0.03 & $1.15(1.07-1.24)$ & $<0.001$ \\
\hline Door-to-needle $\times$ COVID-19 period & & & $0.97(0.83-1.13)$ & 0.65 \\
\hline Age, per decade & $1.72(1.39-2.12)$ & $<0.001$ & $1.35(1.11-1.64)$ & $<0.001$ \\
\hline Female sex & $1.36(0.80-2.29)$ & 0.25 & & \\
\hline White race & $2.20(1.06-4.56)$ & 0.03 & $2.09(0.98-4.45)$ & 0.056 \\
\hline Hispanic ethnicity & $0.97(0.56-1.70)$ & 0.92 & & \\
\hline Transfer from outside hospital & $1.14(0.26-5.03)$ & 0.87 & & \\
\hline Hypertension & $1.07(0.58-1.96)$ & 0.83 & & \\
\hline Dyslipidemia & $1.02(0.61-1.73)$ & 0.93 & & \\
\hline Diabetes & $1.07(0.62-1.85)$ & 0.80 & & \\
\hline Coronary artery disease & $1.35(0.72-2.54)$ & 0.35 & & \\
\hline Prior ischemic stroke & $1.16(0.61-2.22)$ & 0.65 & & \\
\hline Atrial fibrillation & $3.14(1.80-5.50)$ & $<0.001$ & $1.22(0.75-1.99)$ & 0.41 \\
\hline Tobacco use & $0.44(0.15-1.23)$ & 0.12 & & \\
\hline Heart failure & $2.14(1.08-4.23)$ & 0.03 & $1.77(0.65-4.82)$ & 0.27 \\
\hline NIHSS, per point & $1.15(1.11-1.19)$ & $<0.001$ & $1.15(1.12-1.17)$ & $<0.001$ \\
\hline Large vessel occlusion & $1.58(0.90-2.78)$ & 0.11 & & \\
\hline $\mathrm{sICH}^{\mathrm{b}}$ & $8.43(3.81-18.63)$ & $<0.001$ & $8.06(4.14-15.70)$ & $<0.001$ \\
\hline
\end{tabular}

OR denotes odds ratio (with 95\% confidence interval), COVID-19 coronavirus disease 2019, NIHSS National Institutes of Health Stroke Scale, and $s I C H$ symptomatic intracerebral hemorrhage within $36 \mathrm{~h}$ of thrombolysis

${ }^{a}$ Final multivariable model shown is inclusive of the interaction term for treatment delay on admission during the COVID-19 period (AIC 284.28)

b sICH was defined using NINDS criteria as any neurologic worsening attributed to an intracranial hemorrhage which occurred within $36 \mathrm{~h}$ of thrombolysis in discharge to hospice or in-hospital death among patients admitted before or during the COVID-19 pandemic, as we have shown in a prior analysis with greater statistical power, [6] these treatment delays occurred more frequently during the pandemic. Therefore, one may deduce that the greater treatment delays during the pandemic are contributing to worse outcomes among patients treated during this period.

The relationship between time and brain has been well characterized, [8] and our study confirms that even small treatment delays can lead to significant short-term consequences for these patients [6]. Identification of the specific stroke workflow components that could potentially impact such delays associated with reperfusion therapy would be crucial in improving the stroke metrics and their associated outcomes. Delays in arrival to imaging as was seen in a study by $\mathrm{Wu}$ et al. could be contributory although interestingly the same effect was not seen in our study and a few others [9-11]. This could be postulated in part due to the drastic reduction of imaging modality use during the pandemic period with almost $39 \%$ reduction as was noted in a study by Kansagra et al. that possibly led to increased resource availability and resulting efficiency [12]. In this setting, delays from imaging to thrombolysis, are the primary area of contention that are yet to be elucidated, and the major factors associated with treatment delays overall. Future studies targeting the imaging to thrombolysis component of acute stroke workflows might play a vital role in helping reduce treatment delays and thereby improve outcomes in this continuing pandemic.

\section{Limitations}

Although the effect estimate of our findings and the performance of our models were robust, our study remains limited by its retrospective nature and small sample size. It is possible that the small sample size may have contributed to a type II error in our assessment of the interaction between treatment delay and the COVID-19 admission period. We have previously shown that small, but non-significant increases in-hospital mortality among stroke patients admitted during COVID-19 are likely mediated by a greater severity of the stroke [13]. It remains possible that admission during COVID-19 may be an independent risk factor for early mortality and unfavorable short-term outcomes. Larger 
observational studies or pooling of published cohorts may reveal this association. Our study is also limited by the lack of available data regarding concomitant COVID-19 diagnoses among patients admitted during the COVID-19 period, which could have also contributed to higher mortality/ hospice during the COVID-19 period. That said, infection rates among all consecutively hospitalized stroke patients are $<5 \%$, and while mortality is high among COVID-19 stroke patients $(\sim 20-40 \%)$, the overall small proportion of COVID-19 stroke patients is not likely to contribute meaningfully to the present analysis [6]. Further, there was no significant increase in mortality/hospice in this nested cohort study.

\section{Conclusion}

The data from our study indicate that stroke treatment delays during the COVID-19 pandemic can lead to significant short-term consequences for stroke patients. Because treatment delays appear more common during the COVID-19 period, stroke centers should evaluate local practice paradigms to expedite the acute care of stroke patients during COVID-19-and any crisis for that matter- to reduce the odds of short-term unfavorable outcomes.

Funding None.

\section{Declarations}

Conflicts of interest RGN reports consulting fees for advisory roles with Anaconda, Biogen, Cerenovus, Genentech, Imperative Care, Medtronic, Phenox, Prolong Pharmaceuticals, Stryker Neurovascular and stock options for advisory roles with Astrocyte, Brainomix, Cerebrotech, Ceretrieve, Corindus Vascular Robotics, Vesalio, Viz-AI, and Perfuze. No other authors report any competing financial interests.

\section{References}

1. Uchino K, Kolikonda MK, Brown D et al (2020) Decline in stroke presentations during COVID-19 surge. Stroke 51(8):2544-2547

2. Solomon MD, McNulty EJ, Rana JS et al (2020) The Covid-19 pandemic and the incidence of acute myocardial infarction. $\mathrm{N}$ Engl J Med 383(7):691-693

3. Jillella DV, Janocko NJ, Nahab F et al (2020) Ischemic stroke in COVID-19: an urgent need for early identification and management. PLoS ONE 15(9):e0239443

4. Ortega-Gutierrez S, Farooqui M, Zha A et al (2021) Decline in mild stroke presentations and intravenous thrombolysis during the COVID-19 pandemic: The Society of Vascular and Interventional Neurology Multicenter Collaboration. Clin Neurol Neurosurg 201:106436

5. Kamal N, Sheng S, Xian Y et al (2017) Delays in door-to-needle times and their impact on treatment time and outcomes in get with the guidelines-stroke. Stroke 48(4):946-954

6. Siegler JE, Zha AM, Czap AL et al (2021) Influence of the COVID-19 pandemic on treatment times for acute ischemic stroke: The Society of Vascular and Interventional Neurology Multicenter Collaboration. Stroke 52(1):40-47

7. Group TNt-PSS (1997) Intracerebral hemorrhage after intravenous t-PA therapy for ischemic stroke. Stroke 28(11):2109-2118

8. Saver JL (2006) Time is brain-quantified. Stroke 37(1):263-266

9. Wu Y, Chen F, Wang Z et al (2020) Reductions in hospital admissions and delays in acute stroke care during the pandemic of COVID-19. Front Neurol 11:584734

10. Neves Briard J, Ducroux C, Jacquin G et al (2021) Early impact of the COVID-19 pandemic on acute stroke treatment delays. Can J Neurol Sci 48(1):122-126

11. Padmanabhan N, Natarajan I, Gunston R, Raseta M, Roffe C (2021) Impact of COVID-19 on stroke admissions, treatments, and outcomes at a comprehensive stroke centre in the United Kingdom. Neurol Sci 42(1):15-20

12. Kansagra AP, Goyal MS, Hamilton S, Albers GW (2020) Collateral effect of Covid-19 on stroke evaluation in the United States. N Engl J Med. https://doi.org/10.1056/NEJMc2014816

13. Siegler JE, Heslin ME, Thau L, Smith A, Jovin TG (2020) Falling stroke rates during COVID-19 pandemic at a comprehensive stroke center. J Stroke Cerebrovasc Dis 29(8):104953

\section{Authors and Affiliations}

Dinesh V. Jillella ${ }^{1}\left[\right.$ - Fadi Nahab ${ }^{1} \cdot$ Thanh N. Nguyen $^{2} \cdot$ Mohamad Abdalkader $^{3} \cdot$ David S. Liebeskind $^{4} \cdot$ Nirav Vora $^{5}$. Vivek Rai ${ }^{5}$. Diogo C. Haussen ${ }^{6}$ - Raul G. Nogueira ${ }^{6}$. Shashvat Desai ${ }^{7,8}$. Ashutosh P. Jadhav ${ }^{7,8}$. Alexandra L. Czap ${ }^{9}$. Alicia M. Zha ${ }^{9}$. Italo Linfante ${ }^{10}$. Ameer E Hassan ${ }^{11}$. Darko Quispe-Orozco ${ }^{12}$. Santiago Ortega-Gutierrez ${ }^{12}$. Priyank Khandelwal ${ }^{13} \cdot$ Pratit Patel $^{13}$. Osama Zaidat ${ }^{14} \cdot$ Tudor G. Jovin $^{15} \cdot$ Scott Kamen $^{16}$. James E. Siegler ${ }^{15}$

1 Department of Neurology, Emory University School of Medicine, Atlanta, GA 30322, USA

2 Interventional Neurology and Neuroradiology, Boston Medical Center, Boston, MA 02118, USA

3 Department of Neurology, Boston Medical Center, Boston, MA 02118, USA

4 Department of Neurology, Ronald Reagan University of California at Los Angeles, Los Angeles, CA 90095, USA
5 OhioHealth Neuroscience Center, Riverside Methodist Hospital, Columbus, OH 43214, USA

6 Marcus Stroke and Neuroscience Center, Grady Memorial Hospital, Atlanta, GA 30303, USA

7 University of Pittsburgh Medical Center Mercy Hospital, Pittsburgh, PA 15219, USA

8 University of Pittsburgh Medical Center Presbyterian Medical Center, Pittsburgh, PA 15213, USA 
9 Department of Neurology, University of Texas McGovern Medical School, Houston, TX 77030, USA

10 Department of Interventional Neuroradiology and Endovascular Neurosurgery, Baptist Health South Florida, Coral Gables, FL 33146, USA

11 Department of Neurology, University of Texas Rio Grande Valley, Valley Baptist Medical Center, Harlingen, TX 78550, USA

12 Department of Neurology, Neurosurgery and Radiology, University of Iowa Hospitals and Clinics, Iowa City, IA 52242, USA
13 Department of Endovascular Neurological Surgery and Neurology, Robert Wood Johnson University Hospital, New Brunswick, NJ 08901, USA

14 Department of Neurology, Mercy Health St. Vincent Hospital, Toledo, OH 43608, USA

15 Cooper Neurological Institute, Cooper University Hospital, 3 Cooper Plaza, Suite 320, Camden, NJ 08103, USA

16 Cooper Medical School of Rowan University, Camden, NJ 08103, USA 